\title{
Entangled Quantum Fields near the Event Horizon, and Entropy
}

\author{
A. Iorio ${ }^{a, *}$, G. Lambiase ${ }^{b, c, \dagger}$, G.Vitiello ${ }^{b, c, d, \ddagger}$ \\ ${ }^{a}$ Center for Theoretical Physics, Massachusetts Institute of Technology, \\ Cambridge, MA 02139-4307, USA \\ and INFN, Rome, Italy \\ ${ }^{b}$ Dipartimento di Fisica "E.R.Caianiello" Università di Salerno, 84081 Baronissi (SA), Italy \\ ${ }^{c}$ INFN, Gruppo Collegato di Salerno \\ ${ }^{d}$ INFM, Salerno, Italy
}

(October 28, 2018)

\begin{abstract}
By fully exploiting the existence of the unitarily inequivalent representations of quantum fields, we exhibit the entanglement between inner and outer particles, with respect to the event horizon of a black hole. We compute the entanglement entropy and we find that the nonunitarity of the mapping, between the vacua in the flat and the curved frames, makes the entanglement very robust.
\end{abstract}

PACS No.: 03.65.Ud; 04.62.+v; 04.70.Dy

Keyword(s): Entanglement and Quantum Nonlocality; QFT in Curved Space-Times; Quantum Aspects of Black Holes, Evaporation, Thermodynamics

MIT-CTP 3399

\footnotetext{
*E-mail: iorio@lns.mit.edu

†E-mail: lambiase@sa.infn.it

${ }^{\ddagger}$ E-mail: vitiello@sa.infn.it
} 


\section{INTRODUCTION}

Black hole quantum physics nowadays is the most promising theoretical laboratory to test fundamental ideas about nature. Some of the most fascinating issues arising in the struggle to understand black hole quantum physics are: the origin of the entropy of a black hole, the holographic paradigm, the paradox of the information loss. All of them are related to what seems to be a deep and fundamental problem: the counting of the relevant degrees of freedom. Of course, all those paradoxes and difficulties originate from the absence of a reliable quantum theory of gravity. Hence a better understanding of them could shed some light on the long-standing problem of quantizing gravity.

By using thermodynamical arguments, Bekenstein obtained that the entropy of a black hole is proportional to the area of the event horizon [1],

$$
S \sim \mathcal{A}
$$

and not to the volume, as for ordinary matter. Bekenstein's derivation left open the crucial question of the microscopic explanation of such a result. There are, of course, many approaches aimed to explain the microscopic origin of the black hole entropy in several contexts. In the framework of string theory, the microscopic derivation has been pioneered by Strominger and Vafa [2], see also Ref. [3] for a general review, and Refs. [4-7]. On the other hand, the problem has been faced also by considering the value of the Euclidean action [8], the rate of pair creation of black holes [9], the Noether charge associated with a bifurcating Killing horizon [10].

By considering a quantum scalar field in the presence of the gravitational field, treated as a classical background described by general relativity [11], Hawking showed that quantum effects lead to thermal evaporation of black holes [12], with a temperature given by

$$
T=\frac{\kappa}{2 \pi}
$$

where $\kappa=(2 G M)^{-1}$ is the surface gravity of the black hole (through the paper we shall use the natural units $\hbar=c=k_{B}=1$ ).

Hawking effect seems to be more fundamental than the theory within which it has been derived (see for instance [3,13]), nonetheless it is surely associated with the existence of an horizon in the space-time, thus Schwarzschild and other background space-times have been extensively investigated. A case in point is the Rindler space-time [14], that is a flat space-time with an horizon, associated with a uniformly accelerated observer in Minkowski space-time [15-17]. A basic point in Hawking's original proposal [12], is that the evaporation of black holes through quantum radiation induces nonunitary evolution of quantum fields. This gives raise in turn to the intriguing puzzle that information can be lost. The traditional quantum field theoretical approach is expected to fail to describe these phenomena, being based on the unitarity. In other words, if black holes' evaporation has to be described in quantum field theory, then the request of unitarity, deeply rooted in the conventional approach, has to be relaxed.

In this paper we shall show that the existence of the unitarily inequivalent representations of quantum fields allows to calculate, in a new setting, the entanglement entropy of black 
holes. An unexpected consequence of the nonunitarity of the mapping among the vacua in the flat and curved frames, is the robustness of the entanglement between scalar quantum modes living on the two space-like separated sides of the event horizon.

Our approach is based on the construction of an entropy operator directly from the condensate structure of the quantum vacuum. The main steps involved are two: first, we realize that the vacuum state of a Minkowskian observer $\left|0_{M}\right\rangle$ is seen as a condensate of entangled modes by a generic observer, and there is no unitary transformation to disentangle $\left|0_{M}\right\rangle$; second, we construct the von Neumann entropy operator from this vacuum and investigate its properties.

Some preliminary results in this direction already appeared while investigating more general features of field quantization in curved background $[18,19]$. Here we shall fully exploit the entropy operator and the nonunitary nature of the entanglement to compute the entanglement entropy of a black hole in this field theoretical setting.

The entanglement entropy of black hole has been long investigated [20-23]. However, our present study shows that the nonunitarity of the mapping between the vacua in the flat and curved frames is the root of the entanglement robustness.

The paper is organized as follows: In Sec. II we recall the main features of the quantization of a complex scalar field in curved space-time. In Sec. III we analyze the structure of the quantum vacuum, we show that it is a condensate of infinitely many entangled particles living in the two space-like separated regions, and we comment on the nonunitarity of the mapping between the vacua in the flat and the curved frames. In Sec. IV the entropy operator is eventually constructed and studied. We then show in which sense its correct vacuum expectation is the entangled entropy. The application of the obtained results to the Schwarzschild space-time is the argument of Sec. V. Conclusions are drawn in Sec. VI. In Appendix A we present some details of the derivation of the entropy operator, and in Appendix B the Rindler space-time is investigated.

\section{QUANTIZATION OF SCALAR FIELDS IN CURVED SPACE-TIME}

In this Section we recall the well known main steps of the quantization of scalar fields in a curved background $[11,12,15-19]$, in order to develop the formalism to derive the entanglement and the entropy.

Consider a complex massive scalar quantum field $\phi(x)$ in the $D$-dimensional Minkowski space-time, with Lagrangian density

$$
\mathcal{L}\left(\phi^{*}, \phi\right)=\partial_{\mu} \phi^{*} \partial^{\mu} \phi-m^{2} \phi^{*} \phi .
$$

As usual, $\phi(x)$ can be decomposed in Minkowski modes $\left\{U_{k}(x)\right\}$, orthonormal with respect to the Klein-Gordon inner product defined as $\left(\phi_{1}, \phi_{2}\right)=i \int \phi_{1}^{*} \overleftrightarrow{\partial_{t}} \phi_{2} d^{D-1} x$. Let us keep the $(D-1)$-volume finite, and use periodic boundary conditions $k_{n}^{i}=2 \pi L_{i}^{-1} n_{i}$, with $i=1, \ldots, D$, $n_{i}$ integers, and the volume given by $L_{1} \cdot \ldots \cdot L_{D}$, to write

$$
\phi(x)=\sum_{k}\left[a_{k} U_{k}(x)+\bar{a}_{k}^{\dagger} U_{k}^{*}(x)\right],
$$

with $k=\left(k_{1}, \vec{k}\right)$. The quantum Hamiltonian is then given by 


$$
H_{M}=\sum_{k} \omega_{k}\left(a_{k}^{\dagger} a_{k}+\bar{a}_{k}^{\dagger} \bar{a}_{k}\right)
$$

where $\omega_{k}=\sqrt{k_{1}^{2}+|\vec{k}|^{2}+m^{2}}$, and $a_{k}, a_{k}^{\dagger}\left(\bar{a}_{k}, \bar{a}_{k}^{\dagger}\right)$ are the annihilation and creation operators, respectively, for particles (antiparticles). They act on the Hilbert-Fock space $\mathcal{H}$, the Minkowski vacuum being defined by

$$
a_{k}\left|0_{M}\right\rangle=\bar{a}_{k}\left|0_{M}\right\rangle=0, \quad \forall k .
$$

and satisfy the usual canonical commutation relations (CCRs)

$$
\left[a_{k}, a_{k^{\prime}}^{\dagger}\right]=\delta_{k k^{\prime}}, \quad\left[\bar{a}_{k}, \bar{a}_{k^{\prime}}^{\dagger}\right]=\delta_{k k^{\prime}} .
$$

Let us now recall the key features of the quantization of the same scalar field $\phi(x)$ in curved space-times. One expands the field in terms of the complete set (with respect to KleinGordon product) of functions $\left\{u_{p}^{(\sigma)}(x)\right\}$, where $p=(\Omega, \vec{k})$, and the symbol $\sigma= \pm$ takes into account the fact that the space-time has an horizon, so that it is divided into two causally disconnected regions: the internal and external regions of the Schwarzschild geometry, the two Rindler wedges, etc.. The functions $\left\{u_{p}^{(\sigma)}(x)\right\}$ are derived by solving the Klein-Gordon equation in the coordinates of space-time under consideration. Thus, one writes

$$
\phi(x)=\sum_{\sigma} \sum_{p}\left[b_{p}^{(\sigma)} u_{p}^{(\sigma)}(x)+\bar{b}_{p}^{(\sigma) \dagger} u_{p}^{(\sigma) *}(x)\right],
$$

where the operators $b_{p}^{(\sigma)}$ and $\bar{b}_{p}^{(\sigma)}$ are assumed to satisfy the usual CCRs.

By introducing the operators [17]

$$
d_{p}^{(\sigma)}=\sum_{k_{1}} \mathcal{P}_{\Omega}^{(\sigma)}\left(k_{1}\right) a_{k_{1} \vec{k}}
$$

and similarly for $\bar{d}_{p}^{(\sigma)}$ in terms of $\bar{a}_{k}$, where $\left\{\mathcal{P}_{\Omega}^{(\sigma)}\left(k_{1}\right)\right\}$ is a complete set of orthogonal functions, the operators $b_{p}^{(\sigma)}$, and $\bar{b}_{p}^{(\sigma)}$ can be expressed in terms of the Bogolubov transformations $[11,17]$

$$
\begin{aligned}
b_{p}^{(\sigma)} & =d_{p}^{(\sigma)} \cosh \epsilon(p)+\bar{d}_{\tilde{p}}^{(-\sigma) \dagger} \sinh \epsilon(p)=G(\epsilon) d_{p}^{(\sigma)} G^{-1}(\epsilon), \\
\bar{b}_{\tilde{p}}^{(-\sigma) \dagger} & =d_{p}^{(\sigma)} \sinh \epsilon(p)+\bar{d}_{\tilde{p}}^{(-\sigma) \dagger} \cosh \epsilon(p)=G(\epsilon) \bar{d}_{\tilde{p}}^{(-\sigma)} G^{-1}(\epsilon),
\end{aligned}
$$

where $\tilde{p}=(\Omega,-\vec{k})$, and the generator of the transformations is

$$
G(\epsilon)=\exp \left\{\sum_{\sigma} \sum_{p} \epsilon(p)\left[d_{p}^{(\sigma)} \bar{d}_{\tilde{p}}^{(-\sigma)}-d_{p}^{(\sigma) \dagger} \bar{d}_{\tilde{p}}^{(-\sigma) \dagger}\right]\right\} .
$$

At finite volume $G(\epsilon)$ is a unitary operator:

$$
G^{-1}(\epsilon)=G(-\epsilon)=G^{\dagger}(\epsilon) .
$$

The canonical operators $d_{p}^{(\sigma)}$ and $\bar{d}_{p}^{(\sigma)}$ annihilate the Minkowski vacuum $\left|0_{M}^{(+)}\right\rangle \otimes\left|0_{M}^{(-)}\right\rangle$. On the other hand, the operators $b_{p}^{(\sigma)}$, and $\bar{b}_{p}^{(\sigma)}$ annihilate the vacuum 


$$
\left|0^{(+)}(\epsilon)\right\rangle \otimes\left|0^{(-)}(\epsilon)\right\rangle=G(\epsilon)\left[\left|0_{M}^{(+)}\right\rangle \otimes\left|0_{M}^{(-)}\right\rangle\right]
$$

In what follows we shall keep the short-hand notation: $\left|0_{M}\right\rangle \equiv\left|0_{M}^{(+)}\right\rangle \otimes\left|0_{M}^{(-)}\right\rangle$, and $|0(\epsilon)\rangle \equiv$ $\left|0^{(+)}(\epsilon)\right\rangle \otimes\left|0^{(-)}(\epsilon)\right\rangle$.

The parameter $\epsilon$ in Eqs. (II.7) and (II.8) is given by

$$
\sinh \epsilon(p)=\frac{1}{\left(e^{\Omega / T}-1\right)^{1 / 2}},
$$

where $T$ is related to the surface gravity of black holes, in the case of Schwarzschild geometry [12], or to the acceleration, in the case of Rindler geometry [16]. One can also show that the total Hamiltonian is given by $[11,12,15-17,19,24]$

$$
\begin{aligned}
H_{\epsilon} & =\sum_{\sigma} \sum_{p} \sigma \Omega\left[b_{p}^{(\sigma) \dagger} b_{p}^{(\sigma)}+\bar{b}_{\tilde{p}}^{(\sigma)} \bar{b}_{\tilde{p}}^{(\sigma) \dagger}\right] \\
& =H^{(+)}(\epsilon)-H^{(-)}(\epsilon)
\end{aligned}
$$

where we chose not to normal-order, as some considerations on the entropy will be clearer in this setting (see Sec. V). Let us also note that the vacuum $|0(\epsilon)\rangle$ does depend on $T$, hence depends on the physical parameters that characterize the background.

The Bogolubov transformations, Eqs. (II.7) and (II.8), relate the vectors of $\mathcal{H}=\mathcal{H}^{(+)} \otimes \mathcal{H}^{(-)}$ to the vectors of another Hilbert-Fock space $\mathcal{H}_{\epsilon}=\mathcal{H}_{\epsilon}^{(+)} \otimes \mathcal{H}_{\epsilon}^{(-)}$labelled by $\epsilon$. In our approach the structure of the vacuum, and of course of the Hilbert space, is crucial. We shall dedicate the next Section to the investigation of their physical properties.

\section{VACUUM STRUCTURE AND ENTANGLEMENT}

The relation between the spaces $\mathcal{H}$ and $\mathcal{H}_{\epsilon}$ is established by the generator $G(\epsilon): \mathcal{H} \rightarrow \mathcal{H}_{\epsilon}$, or by its inverse $G^{-1}(\epsilon): \mathcal{H}_{\epsilon} \rightarrow \mathcal{H}$. The physical meaning of this freedom is that we can arbitrarily choose to express Minkowskian quantities in terms of generic $\epsilon$-quantities, or the other way around. We choose, for instance, to express the Minkowskian vacuum in terms of the generic $\epsilon$-vacuum

$$
\left|0_{M}\right\rangle=G^{-1}(\epsilon)|0(\epsilon)\rangle .
$$

This relation holds only at finite degrees of freedom, i.e. finite volume. Note that $G(\epsilon)$ is an element of ${ }^{1} S U(1,1) \times S U(1,1)$. Of course the same structure arises by writing $G^{-1}(\epsilon)$ in terms of the $b \mathrm{~s}$, all one has to do is to replace $d \rightarrow b$ in the Eq. (II.9). Thus by

\footnotetext{
${ }^{1}$ To be more precise, for each mode, we have the direct product of two two-boson realizations of $S U(1,1):\left[T_{+}^{i}, T_{-}^{i}\right]=-2 T_{0}^{i},\left[T_{0}^{i}, T_{ \pm}^{i}\right]= \pm T_{ \pm}^{i}, i=1,2$, where $\left[T^{1}, T^{2}\right]=0$, for all the generators $T$. This is seen by defining $T_{+}^{1}=d^{(+) \dagger} \bar{d}^{(-) \dagger}, T_{-}^{1}=d^{(+)} \bar{d}^{(-)}, T_{0}^{1}=\frac{1}{2}\left(d^{(+) \dagger} d^{(+)}+\bar{d}^{(-) \dagger} \bar{d}^{(-)}\right)$, while $T_{+}^{2}$, $T_{-}^{2}, T_{0}^{2}$ are obtained by replacing $(+) \leftrightarrow(-)$.
} 
using the Gaussian decomposition, the Minkowski vacuum can be formally expressed as a $S U(1,1) \times S U(1,1)$ generalized coherent state [25] of Cooper-like pairs

$$
\left|0_{M}\right\rangle=\frac{1}{Z} \exp \left[\sum_{\sigma} \sum_{p} \tanh \epsilon(p) b_{p}^{(\sigma) \dagger} \bar{b}_{\tilde{p}}^{(-\sigma) \dagger}\right]|0(\epsilon)\rangle
$$

where $Z=\prod_{p} \cosh ^{2} \epsilon(p)$.

In the continuum limit in the space of momenta, i.e. in the infinite-volume limit, the number of degrees of freedom becomes uncountable infinite, hence we have

$$
\begin{aligned}
\left\langle 0(\epsilon) \mid 0_{M}\right\rangle \rightarrow 0 \quad \text { as } \quad V \rightarrow \infty, & \forall \epsilon \\
\left\langle 0(\epsilon) \mid 0\left(\epsilon^{\prime}\right)\right\rangle \rightarrow 0 \quad \text { as } \quad V \rightarrow \infty, & \forall \epsilon, \epsilon^{\prime}, \epsilon \neq \epsilon^{\prime},
\end{aligned}
$$

where $V$ is the volume of the whole $(D-1)$-dimensional space. This means that the Hilbert spaces $\mathcal{H}$ and $\mathcal{H}_{\epsilon}$ become unitarily inequivalent in the continuum limit. In this limit $\epsilon$ labels the set $\left\{H_{\epsilon}, \forall \epsilon\right\}$ of the infinitely many unitarily inequivalent representations of the CCRs $[24,26]$.

Let us now discuss the entanglement of the vacuum $\left|0_{M}\right\rangle$ in (III.2), that we rewrite in the following convenient form

$$
\left|0_{M}\right\rangle=\frac{1}{Z}\left[|0(\epsilon)\rangle+\sum_{p} \tanh \epsilon(p)\left(\left|1_{p}^{(+)}, \overline{0}\right\rangle \otimes\left|0, \overline{1}_{\tilde{p}}^{(-)}\right\rangle+\left|0, \overline{1}_{\tilde{p}}^{(+)}\right\rangle \otimes\left|1_{p}^{(-)}, \overline{0}\right\rangle\right)+\ldots\right]
$$

where, we denote by $\left|n_{p}^{(\sigma)}, \bar{m}_{\tilde{p}}^{(\sigma)}\right\rangle$ a state of $n$ particles and $m$ antiparticles in whichever sector $(\sigma)$. Note that for the generic $n^{\text {th }}$ term, the state $\left|n_{p}^{(\sigma)}, \overline{0}\right\rangle \equiv\left|1_{p_{1}}^{(\sigma)}, \ldots, 1_{p_{n}}^{(\sigma)}, \overline{0}\right\rangle$, and similarly for antiparticles.

By introducing a well-known notation, $\uparrow$ for a particle, and $\downarrow$ for an antiparticle, the twoparticle state in (III.5) can be written as

$$
\left|\uparrow^{(+)}\right\rangle \otimes\left|\downarrow^{(-)}\right\rangle+\left|\downarrow^{(+)}\right\rangle \otimes\left|\uparrow^{(-)}\right\rangle
$$

which is an entangled state of particle and antiparticle living in the two causally disconnected regions $( \pm)$. The generic $n^{\text {th }}$ term in (III.5) shares exactly the same property as the twoparticle state, but this time the $\uparrow$ describes a set of $n$ particles, and $\downarrow$ a set of $n$ anti-particles. The mechanism of the entanglement, dynamically induced by gravitational effects, takes place at all orders in the expansion, always by grouping particles and antiparticles into two sets. Thus the whole vacuum $\left|0_{M}\right\rangle$ is an infinite superposition of entangled states ${ }^{2}$

$$
\left.\left|0_{M}\right\rangle=\sum_{n=0}^{+\infty} \sqrt{W_{n}} \mid \text { Entangled }\right\rangle_{n}
$$

where

\footnotetext{
${ }^{2} \mathrm{~A}$ similar structure also arises in the temperature-dependent vacuum of Thermo-Field Dynamics [24], see also Ref. [27].
} 


$$
W_{n}=\prod_{p} \frac{\sinh ^{2 n_{p}} \epsilon(p)}{\cosh ^{2\left(n_{p}+2\right)} \epsilon(p)},
$$

with

$$
0<W_{n}<1 \text { and } \quad \sum_{n=0}^{+\infty} W_{n}=1
$$

Details of the computations can be found in the Appendix A.

Of course, the probability of having entanglement of two sets of $n$ particles and $n$ antiparticles is $W_{n}$. At finite volume, being $W_{n}$ a decreasing monotonic function of $n$, the entanglement is suppressed for large $n$. It appears then, that only a finite number of entangled terms in the expansion (III.7) is relevant. Nonetheless this is only true at finite volume (the quantum mechanical limit), while the interesting case occurs in the infinite volume limit, which one has to perform in a quantum field theoretical setting.

The entanglement is generated by $G(\epsilon)$, where the scalar field modes in one sector $(\sigma)$ are coupled to the modes in the other sector $(-\sigma)$ via the parameter $\epsilon(p)$, which takes into account the effects of the background gravitational field (environment) [19]. We stress that the origin of the entanglement is the environment, in contrast with the usual quantum mechanical view, which attributes to the environment the loss of the entanglement. In the present treatment such an origin for the entanglement makes it quite robust.

One further reason for the robustness is that this entanglement is realized in the limit to the infinite volume once and for all, since then there is no unitary evolution to disentangle the vacuum: at infinite volume one cannot "unknot the knots". Such a nonunitarity is only realized when all the terms in the series (III.7) are summed up, which indeed happens in the $V \rightarrow \infty$ limit.

Summarizing, the interaction with the environment and nonunitarity, are the basis for the generation and the stability of the entanglement. These two features are entirely due to the nature of the quantum field theoretical vacuum.

\section{THE ENTROPY OPERATOR}

It is now matter of constructing thermodynamical quantities out of the condensate structure of the entangled vacuum $\left|0_{M}\right\rangle$. By using Eqs. (II.7) and (II.8) one obtains that the number of modes of the type $b_{p}^{(\sigma)}$ in $\left|0_{M}\right\rangle$ is given by

$$
\mathcal{N}_{b}^{(\sigma)} \equiv\left\langle 0_{M}\left|b_{p}^{(\sigma) \dagger} b_{p}^{(\sigma)}\right| 0_{M}\right\rangle=\sinh ^{2} \epsilon(p), \quad \sigma= \pm
$$

and similarly for the modes of type $\bar{b}_{\tilde{p}}^{(\sigma)}$.

Algebraic manipulations allow to recast $\left|0_{M}\right\rangle$ in Eq. (III.2) in the form

$$
\begin{aligned}
\left|0_{M}\right\rangle & =e^{-S^{(+)}(\epsilon) / 2} e^{\sum_{p, \sigma} b_{p}^{(\sigma) \dagger} \bar{b}_{\tilde{p}}^{(-\sigma) \dagger}}|0(\epsilon)\rangle \\
& =e^{-S^{(-)}(\epsilon) / 2} e^{\sum_{p, \sigma} b_{p}^{(\sigma) \dagger} \bar{b}_{\tilde{p}}^{(-\sigma) \dagger}}|0(\epsilon)\rangle,
\end{aligned}
$$

where $S^{(+)}(\epsilon)$ and $S^{(-)}(\epsilon)$ are given by 


$$
\begin{aligned}
S^{(+)}(\epsilon)= & \mathcal{S}^{(+)}(\epsilon)+\overline{\mathcal{S}}^{(+)}(\epsilon) \\
= & -\sum_{p}\left[b_{p}^{(+) \dagger} b_{p}^{(+)} \ln \sinh ^{2} \epsilon(p)-b_{p}^{(+)} b_{p}^{(+) \dagger} \ln \cosh ^{2} \epsilon(p)\right. \\
& +(b \rightarrow \bar{b})], \\
S^{(-)}(\epsilon)= & \mathcal{S}^{(-)}(\epsilon)+\overline{\mathcal{S}}^{(-)}(\epsilon) \\
= & -\sum_{p}\left[b_{p}^{(-) \dagger} b_{p}^{(-)} \ln \sinh ^{2} \epsilon(p)-b_{p}^{(-)} b_{p}^{(-) \dagger} \ln \cosh ^{2} \epsilon(p)\right. \\
& +(b \rightarrow \bar{b})] .
\end{aligned}
$$

The formulae (IV.2) will be proved in some details in the Appendix A.

One can easily convince himself that $S^{(\sigma)}(\epsilon), \sigma= \pm$, is nothing else than the von Neumann entropy

$$
S=-\mathcal{N} \ln \mathcal{N},
$$

where $\mathcal{N}$ is the number of microscopic states. At the origin of this entropy there are the vacuum fluctuations of the quantum states, which have a thermal character for different observers related to the Minkowski observer through a diffeomorphism. By counting the number of occupation states in the vacuum $\left|0_{M}\right\rangle$ with the number operator for particles $N_{b}^{(\sigma)} \equiv b_{p}^{(\sigma) \dagger} b_{p}^{(\sigma)}$, we must subtract those occupation states counted by the operator $\bar{b}_{\tilde{p}}^{(\sigma)} \bar{b}_{\tilde{p}}^{(\sigma) \dagger}=1+N_{\bar{b}}^{(\sigma)}$, where $N_{\bar{b}}^{(\sigma)}$ is the number operator for the antiparticles. This accounts for our definitions (IV.3) and (IV.4) of the entropy operators

$$
S^{(\sigma)}(\epsilon)=-\sum_{p}\left[N_{b}^{(\sigma)} \ln \mathcal{N}_{b}^{(\sigma)}-\left(1+N_{\bar{b}}^{(\sigma)}\right) \ln \left(1+\mathcal{N}_{\bar{b}}^{(\sigma)}\right)+(b \rightarrow \bar{b})\right] .
$$

We note that in terms of the coefficients $W_{n}$ in Eq. (III.8) we have

$$
\left\langle 0_{M}\left|S^{(\sigma)}(\epsilon)\right| 0_{M}\right\rangle=-\sum_{n \geq 0} W_{n} \ln W_{n} .
$$

We emphasize that the operator $S^{(+)}(\epsilon)=\mathcal{S}^{(+)}(\epsilon)+\overline{\mathcal{S}}^{(+)}(\epsilon)$ is the sum of the entropy operators for the boson gas of particles and antiparticles in the sector $(+)$, similarly for $S^{(-)}(\epsilon)$ in the sector $(-)$. The total entropy operator is given by

$$
S_{\epsilon}=S^{(+)}(\epsilon)-S^{(-)}(\epsilon),
$$

and, as the Hamiltonian (II.12), it is the difference of the two operators. The Bogolubov transformations leave $S_{\epsilon}$ invariant, $\left[S_{\epsilon}, G(\epsilon)\right]=0$, hence $S_{\epsilon}\left|0_{M}\right\rangle=0$. This means that one can arbitrarily choose one of the two sectors, $\sigma= \pm$, to "measure" the correspondent entropy $S^{( \pm)}(\epsilon)$ relative to the ground state $\left|0_{M}\right\rangle$. Let us work in the sector $\sigma=+$.

Now we want to show in which sense this entropy is an entanglement entropy. When one computes the vacuum expectation value $\left\langle 0(\epsilon)\left|S^{(+)}(\epsilon)\right| 0(\epsilon)\right\rangle$ the result is

$$
\left\langle 0(\epsilon)\left|S^{(+)}(\epsilon)\right| 0(\epsilon)\right\rangle=-2 \sum_{\Omega, \vec{k}} \ln \cosh ^{2} \epsilon(\Omega),
$$


which is divergent due to the infinite sum on the momenta $\sum_{\vec{k}}$. Nevertheless, if the entropy operator is normal-ordered, the term $\ln \cosh ^{2} \epsilon(\Omega)$ is removed and the expectation value of the entropy vanishes.

Nonetheless with $S^{(+)}(\epsilon)$ we have to look at the entropy of the Minkowski vacuum $\left|0_{M}\right\rangle$, and not of $|0(\epsilon)\rangle$. The generic observer sees the Minkowski vacuum as an entangled condensate. Such an observer then will measure $\left\langle 0_{M}\left|S^{(+)}(\epsilon)\right| 0_{M}\right\rangle$. The result of such a "cross measurement" gives

$$
\left\langle 0_{M}\left|S^{(+)}(\epsilon)\right| 0_{M}\right\rangle=-2 \sum_{\Omega, \vec{k}}\left[\sinh ^{2} \epsilon(\Omega) \ln \sinh ^{2} \epsilon(\Omega)-\cosh ^{2} \epsilon(\Omega) \ln \cosh ^{2} \epsilon(\Omega)\right],
$$

where Eq. (IV.1) has been used. We notice that again there is a divergence due to $\sum_{\vec{k}}$, but this time even if the expression is normal-ordered the result is never zero (cfr. e.g. Sec V). The physical meaning of such occurrence is that the entanglement is dynamically generated via the interaction with the gravitational background, as already observed. The entanglement is only seen if the generic observer measures on the Minkowski vacuum. It is precisely in this sense that we say that the black hole entropy is the entanglement entropy.

As already observed, in the $V \rightarrow \infty$ limit the generator $G(\epsilon)$ does not exist, but the whole structure described above survives. What one loses in that limit is the possibility to generate the mapping between the states of the two frames, the Minkowski and the generic one. In some sense, the "symmetry" in writing $\left\langle 0_{M}\left|S^{(+)}(\epsilon)\right| 0_{M}\right\rangle=\left\langle 0(\epsilon)\left|G(\epsilon) S^{(+)}(\epsilon) G^{-1}(\epsilon)\right| 0(\epsilon)\right\rangle=$ $\left\langle 0(\epsilon)\left|S_{M}^{(+)}\right| 0(\epsilon)\right\rangle$ is lost.

For the sake of completeness we conclude this Section by noticing that the "free-energy" can also be introduced [24]

$$
\mathcal{F}^{(+)}(\epsilon) \equiv\left\langle 0_{M}\left|H^{(+)}(\epsilon)-\frac{1}{\beta} S^{(+)}(\epsilon)\right| 0_{M}\right\rangle
$$

It is interesting to note that by looking for the values of $\epsilon(p)$ making $\mathcal{F}^{(+)}(\epsilon)$ stationary, and considering negligible variations of $\beta$ with respect to $\epsilon$ one obtains

$$
\beta \Omega=-\ln \tanh ^{2} \epsilon(p) \Leftrightarrow \sinh ^{2} \epsilon(p)=\frac{1}{e^{\beta \Omega}-1} .
$$

From Eq. (IV.1), it follows that

$$
\mathcal{N}_{b}^{(+)}=\frac{1}{e^{\beta \Omega}-1}
$$

and similarly for $\mathcal{N}_{\bar{b}}^{(+)}$. In this way one consistently recovers (II.11), which is the Bose distribution provided that $T=\beta^{-1}$ is identified with the temperature.

\section{APPLICATIONS TO THE SCHWARZSCHILD SPACE-TIME}

We derive now the thermodynamical properties of black holes described by the Schwarzschild geometry 


$$
d s^{2}=\left(1-\frac{2 G M}{r}\right) d t^{2}-\left(1-\frac{2 G M}{r}\right)^{-1} d r^{2}-r^{2}\left(d \theta^{2}+\sin ^{2} \theta d \varphi^{2}\right)
$$

where the space-time is taken to be 4-dimensional. The event horizon is given by $r_{S}=2 G M$, and the Bekenstein-Hawking temperature (I.2) is $T \simeq r_{S}^{-1}=(2 G M)^{-1}$.

We want to calculate the finite part of the entropy in Eq. (IV.8) by moving to the continuum limit, taken to be formally identical to the Minkowskian one

$$
\sum_{\Omega, \vec{k}} \rightarrow \frac{V}{(2 \pi)^{3}} \int_{0}^{\infty} d \Omega \int d^{2} k,
$$

where $V$ is the 3 -dimensional volume of the whole space-time.

The entropy density is

$$
\begin{aligned}
\left\langle s^{(+)}(\epsilon)\right\rangle_{M} & \equiv \frac{\left\langle 0_{M}\left|S^{(+)}(\epsilon)\right| 0_{M}\right\rangle}{V} \\
& =\frac{-2}{(2 \pi)^{3}} \int_{0}^{\infty} d \Omega\left[\sinh ^{2} \epsilon(\Omega) \ln \sinh ^{2} \epsilon(\Omega)-\cosh ^{2} \epsilon(\Omega) \ln \cosh ^{2} \epsilon(\Omega)\right] \int d^{2} k .
\end{aligned}
$$

By expressing $\epsilon(\Omega)$ as a function of $\Omega$ through Eq. (II.11), one can compute the integral in Eq. (V.3) to obtain (see Eqs. 3.411-1 and 4.223-2 of Ref. [28])

$$
\left\langle s^{(+)}(\epsilon)\right\rangle_{M}=\frac{\pi^{2} T}{3(2 \pi)^{3}} \int d^{2} k,
$$

which is divergent, as expected. We cannot remove this infinity by normal ordering the entropy operator. Following the recipe adopted in quantum gravity we use a cutoff $k_{C}$ of the order of the Planck momentum $k_{C} \simeq k_{P}=l_{P}^{-1}=G^{-1 / 2}$. Our entropy density is then given by

$$
\left\langle s^{(+)}(\epsilon)\right\rangle_{M}=\frac{k_{C}^{2} T}{24 \pi} .
$$

Being the proper volume in the Schwarzschild geometry

$$
V_{\text {prop }}=\int \sqrt{-g_{r r} g_{\theta \theta} g_{\varphi \varphi}} d r d \theta d \varphi
$$

only defined for $r>r_{S}$, we now have to compute the entropy for the spherical shell of radii $r_{S}$ and $r_{S}+\delta$. The volume of the shell is given by

$$
\mathcal{V}=4 \pi r_{S}^{3} \int_{1}^{1+h} \frac{x^{5 / 2}}{\sqrt{x-1}} d x \propto r_{S}^{3}
$$

where $h=\delta / r_{S}$ is chosen by requiring the numerical factor of proportionality to be $\mathcal{O}(1)$. Since $k_{C} \lesssim k_{P}$, and recalling that the Bekenstein-Hawking temperature is $T \sim r_{S}^{-1}$, we obtain the upper bound for the entropy

$$
\left\langle S^{(+)}(\epsilon)\right\rangle_{M}=\mathcal{V}\left\langle s^{(+)}(\epsilon)\right\rangle_{M} \lesssim \frac{\mathcal{A}}{l_{P}^{2}} .
$$

Hence the entropy is proportional to the horizon area $\mathcal{A}$ of the black hole and is bounded from above. In the Appendix B this analysis will be applied to the Rindler space-time. It is an interesting question to investigate the relation between the derivation of (V.7) and the holographic principle. 


\section{CONCLUSIONS}

We proposed a new method to obtain the entanglement entropy of black holes, based on the relevant nonunitary features of quantum fields. This was done for the Schwarzschild and Rindler (Appendix B) space-times.

Hawking's original proposal [12] that the evaporation of black holes through quantum radiation induces nonunitary evolution of quantum fields was reanalysed. Despite the fact that a description in terms of the traditional (fully unitary) quantum field theoretical approach is then expected to fail, we have shown that the unitarily inequivalent representation of quantum fields allow to calculate, in a new setting, the entanglement entropy of black holes. We have also shown that the entanglement between inner and outer particles, with respect to the event horizon, is very robust due to the nonunitary nature of the mapping between the vacua in the flat and the curved frames.

Our results are obtained assuming a black hole at thermodynamical equilibrium. The approach presented in this paper can be applied to a wide range of problems related to black holes' quantum physics. We shall further exploit this formalism in future works.

\section{ACKNOWLEDGMENTS}

A.I. thanks Roman Jackiw for interesting comments. A.I. and G.V. acknowledge COSLAB Network European Science Foundation. This work is supported in part by funds provided by the U.S. Department of Energy (D.O.E.) under cooperative research agreement DF-FC0294ER40818.

\section{APPENDIX A: Useful Algebraic Relations}

In this Appendix we want to derive the expressions (IV.2) and (III.7) for the vacuum $\left|0_{M}\right\rangle$. We first derive a couple of useful relations. Recalling that $S^{(\sigma)}(\epsilon)=\mathcal{S}^{(\sigma)}(\epsilon)+\overline{\mathcal{S}}^{(\sigma)}(\epsilon)$, we have

$$
\begin{aligned}
e^{-S^{(\sigma)}(\epsilon) / 2} b_{p}^{(\sigma) \dagger} e^{S^{(\sigma)}(\epsilon) / 2} & =b_{p}^{(\sigma) \dagger}+\frac{1}{2}\left[b_{p}^{(\sigma) \dagger}, \mathcal{S}^{(\sigma)}(\epsilon)\right]+\frac{1}{8}\left[\left[b_{p}^{(\sigma) \dagger}, \mathcal{S}^{(\sigma)}(\epsilon)\right], \mathcal{S}^{(\sigma)}(\epsilon)\right]+\ldots \\
& =b_{p}^{(\sigma) \dagger}\left(1+\ln \tanh \epsilon(p)+\frac{1}{2}(\ln \tanh \epsilon(p))^{2}+\ldots\right) \\
& =b_{p}^{(\sigma) \dagger} \exp \{\ln \tanh \epsilon(p)\}=b_{p}^{(\sigma) \dagger} \tanh \epsilon(p)
\end{aligned}
$$

where $\sigma= \pm$. Similarly one can prove the same relation for the antiparticles by using the $\overline{\mathcal{S}}^{(\sigma)}(\epsilon)$ in $S^{(\sigma)}(\epsilon)$ :

$$
e^{-S^{(\sigma)}(\epsilon) / 2} \bar{b}_{p}^{(\sigma) \dagger} e^{S^{(\sigma)}(\epsilon) / 2}=\tanh \epsilon(p) \bar{b}_{p}^{(\sigma) \dagger}
$$

Furthermore, one sees that 


$$
\begin{aligned}
e^{-S^{(\sigma)}(\epsilon) / 2}|0(\epsilon)\rangle & =\exp \left\{\frac{1}{2} \sum_{p}\left[\left(N_{b}^{(\sigma)} \ln \tanh ^{2} \epsilon(p)-\ln \cosh ^{2} \epsilon(p)\right)+(b \rightarrow \bar{b})\right]\right\}|0(\epsilon)\rangle \\
& =\exp \left\{\sum_{p} \ln \tanh \epsilon(p) N_{b}^{(\sigma)}\right\} \exp \left\{-\sum_{p} \ln \cosh \epsilon(p)\right\} \times(b \rightarrow \bar{b})|0(\epsilon)\rangle .
\end{aligned}
$$

By noting that

$$
\begin{aligned}
\exp \left\{\sum_{p} \ln \tanh \epsilon(p) N_{b}^{(\sigma)}\right\}|0(\epsilon)\rangle & =|0(\epsilon)\rangle, \\
\exp \left\{-\sum_{p} \ln \cosh \epsilon(p)\right\}|0(\epsilon)\rangle & =\prod_{p} \cosh ^{-1} \epsilon(p)|0(\epsilon)\rangle,
\end{aligned}
$$

and similarly for antiparticles, one gets

$$
e^{-S^{(\sigma)}(\epsilon) / 2}|0(\epsilon)\rangle=\left[\prod_{p} \cosh ^{-2} \epsilon(p)\right]|0(\epsilon)\rangle
$$

Now we are in the position to prove Eq. (IV.2). Eq. (III.2) can be rewritten as

$$
\left|0_{M}\right\rangle=\prod_{\sigma} \prod_{p}\left(1+\ldots+\frac{1}{n !} \tanh ^{n} \epsilon(p) b_{p}^{(\sigma) \dagger} \bar{b}_{\tilde{p}}^{(-\sigma) \dagger}+\ldots\right)\left[\prod_{p} \cosh ^{-2} \epsilon(p)\right]|0(\epsilon)\rangle,
$$

and by using Eqs. (A.5) and (A.1), one obtains

$$
\left|0_{M}\right\rangle=\left(e^{-S^{(+)} / 2} e^{\sum_{\sigma} \sum_{p} b_{p}^{(\sigma) \dagger} \bar{b}_{\tilde{p}}^{(-\sigma) \dagger}} e^{S^{(+)} / 2}\right)\left[e^{-S^{(+)} / 2}|0(\epsilon)\rangle\right]
$$

similarly with $S^{(-)}$, q.e.d. .

Let us now prove that the coefficients $W_{n}$ in Eq. (III.7) are given by Eq. (III.8). At this end, we recast the vacuum (IV.2) in the form

$$
\begin{aligned}
\left|0_{M}\right\rangle & =e^{-S^{(+)} / 2} \sum_{n_{p}=0}^{\infty}\left[\left|n_{p}^{(+)}, \overline{0}\right\rangle \otimes\left|0, \bar{n}_{\tilde{p}}^{(-)}\right\rangle+\left|0, \bar{n}_{\tilde{p}}^{(+)}\right\rangle \otimes\left|n_{p}^{(-)}, \overline{0}\right\rangle\right] \\
& \equiv e^{-S^{(+)} / 2} \sum_{n_{p}=0}^{\infty} \sum_{\sigma= \pm}\left|n_{p}^{(\sigma)} ; \bar{n}_{\tilde{p}}^{(-\sigma)}\right\rangle
\end{aligned}
$$

where, according to the notation introduced after Eq. (III.5) and in Eq. (III.7), $n=\bar{n}$, and

$$
\left.\sum_{\sigma= \pm}\left|n_{p}^{(\sigma)} ; \bar{n}_{\tilde{p}}^{(-\sigma)}\right\rangle \equiv \mid \text { Entangled }\right\rangle_{n_{p}}
$$

Hence,

$$
\begin{aligned}
\left|0_{M}\right\rangle & =\sum_{n_{p}=0}^{\infty} e^{\sum_{p^{\prime}}\left[n_{p^{\prime}} \ln \sinh \epsilon\left(p^{\prime}\right)-\left(1+n_{p^{\prime}}\right) \ln \cosh \epsilon\left(p^{\prime}\right)\right]} \sum_{\sigma= \pm}\left|n_{p}^{(\sigma)} ; \bar{n}_{\tilde{p}}^{(-\sigma)}\right\rangle \\
& =\sum_{n_{p}=0}^{\infty} \prod_{p} \tanh ^{n_{p}} \epsilon(p) \cosh ^{-1} \epsilon(p) \sum_{\sigma= \pm}\left|n_{p}^{(\sigma)} ; \bar{n}_{\tilde{p}}^{(-\sigma)}\right\rangle \\
& =\sum_{n_{p}=0}^{\infty} \sqrt{W_{n_{p}}} \sum_{\sigma= \pm}\left|n_{p}^{(\sigma)} ; \bar{n}_{\tilde{p}}^{(-\sigma)}\right\rangle
\end{aligned}
$$


which proves Eq. (III.8).

\section{APPENDIX B: The Rindler Space-Time}

In this Appendix we apply the formalism developed in Secs. IV and V to the Rindler space-time [14], corresponding to an observer moving with constant acceleration $a$. This space-time is described by the line element (see for example [11])

$$
d s^{2}=e^{2 a \xi}\left(d \tau^{2}-d \xi^{2}\right)-d y^{2}-d z^{2},
$$

which reduces to Minkowski space-time letting

$$
t=\frac{e^{a \xi}}{a} \sinh a \tau, \quad x=\frac{e^{a \xi}}{a} \cosh a \tau .
$$

This metric covers a portion of Minkowski space-time with $x>|t|$. The boundary planes are determined by $x \pm t=0$ [14]. Davies [15] and Unruh [16] have shown that the vacuum state for an inertial observer is a canonical ensemble for the Rindler observer. The temperature $T_{R}$ characterizing this ensemble is related to the acceleration of the observer by the relation

$$
T_{R}=\frac{a}{2 \pi}
$$

This is the thermalization theorem, in a nutshell (for a review see [17]).

The proper volume is given by

$$
V_{\text {prop }}=\int \sqrt{g_{\xi \xi} g_{y y} g_{z z}} d \xi d y d z=\int_{-\infty}^{0} e^{a \xi} d \xi \int d y d z=\frac{\mathcal{A}}{a},
$$

where $\mathcal{A}=\int d y d z$ is the area of a surface of constant $\xi$ and $\tau$. The entropy density is computed for a cutoff on the momenta $k_{C} \lesssim l_{P}^{-1}$, and is given by

$$
\left\langle s^{(+)}(\epsilon)\right\rangle_{M}=\frac{a k_{C}^{2}}{48 \pi^{2}}
$$

Hence, the entropy computed in the volume $V_{\text {prop }}$, by considering that $T \sim a$ in the Unruh effect (see Eq. (B.3)), is given by

$$
\left\langle S^{(+)}(\epsilon)\right\rangle_{M} \lesssim \frac{\mathcal{A}}{l_{P}^{2}} .
$$

Thus, also in the Rindler case the entropy is proportional to the area of the event horizon (a result also obtained in Ref. [29,22]), and bounded from above.

It is worth to note that in the case of Rindler space-time, results are formally equivalent to the Schwarzschild geometry since the surface gravity of the black hole is the gravitational acceleration at radius $r$ measured at the infinity. 


\section{REFERENCES}

[1] J.D. Bekenstein, Phys. Rev. D 7, 2333 (1973).

[2] A. Strominger, C. Vafa, Phys. Lett. B 379, 99 (1996).

[3] D. Bigatti and L. Susskind, TASI lectures on the holographic principle, arXiv:hepth/0002044.

[4] J. Polchinski, S. Chaudhuri and C. V. Johnson, Notes on D-Branes, arXiv:hepth/9602052.

[5] A. Krause, Schwarzschild Black Holes from Brane-Antibrane Pairs, [arXiv:hepth/0204206]; Dual Brane Pairs, Chains and the Bekenstein-Hawking Entropy, [arXiv:hep-th/0201260].

[6] J.H. Schwarz, Nucl. Phys. B (Proc. Suppl.) 55, 1 (1997).

[7] S. Carlip, Phys. Rev. D 51, 632 (1995) [arXiv:gr-qc/9409052].

A. Strominger, H. High Energy Phys. 02, 009 (1998).

S. Carlip, Phys. Rev. Lett. 82, 2828 (1999).

Mu-In Park, Jeongwon Ho, Phys. Rev. Lett. 83, 5595 (1999).

M. Park, Nucl. Phys. B 634, 339 (2002) [arXiv:hep-th/0111224].

[8] G. W. Gibbons and S. W. Hawking, Phys. Rev. D 15, 2752 (1977).

J.D. Brown, J.M. York, Phys. Rev. D 47, 1420 (1993).

M. Banados, C. Teitelboin, J. Zanelli, Phys. Rev. Lett. 72, 957 (1994).

S.W. Hawking, C.J. Hunter, Phys. Rev. D 59, 044025 (1999).

[9] D. Garfinkle, S. B. Giddings and A. Strominger, Phys. Rev. D 49, 958 (1994) [arXiv:grqc/9306023].

[10] R. M. Wald, Phys. Rev. D 48, 3427 (1993) [arXiv:gr-qc/9307038].

V. Iyer, R.M. Wald, Phys. Rev. D 50, 846 (1994).

S. Mukohyama, The origin of black hole entropy, PhD Thesis Kyoto University, [axXiv:gr-qc/9809050].

[11] N.D. Birrel and P.C.W. Davies, Quantum Fields in Curved Space, Cambridge University Press, Cambridge, 1982.

[12] S.W. Hawking, Commun. Math. Phys. 43, 199 (1975).

[13] M. Visser, Essential and inessential features of Hawking radiation, arXiv:hepth/0106111.

[14] W. Rindler, Essential Relativity, Springer, Berlin, 1977.

[15] P. C. Davies, J. Phys. A 8, 609 (1975).

[16] W. G. Unruh, Phys. Rev. D 14, 870 (1976).

[17] S. Takagi, Progress of Theor. Phys. Suppl. 88 (1986).

[18] M. Martellini, P. Sodano and G. Vitiello, Nuovo Cim. A 48, 341 (1978).

[19] A. Iorio, G. Lambiase and G. Vitiello, Annals Phys. 294, 234 (2001) [arXiv:hepth/0104162].

[20] L. Bombelli, R. K. Koul, J. H. Lee and R. D. Sorkin, Phys. Rev. D 34, 373 (1986).

M. Srednicki, Phys. Rev. Lett. 71, 666 (1993).

V. Frolov, I. Novikov, Phys. Rev. D 48, 4545 (1993)

[21] S. Mukohyama, M. Seriu and H. Kodama, Phys. Rev. D 55, 7666 (1997) [arXiv:grqc/9701059].

[22] H. Terashima, Phys. Rev. D 61, 104016 (2000) [arXiv:gr-qc/9911091]. 
[23] V.P. Frolov, D.V. Fursaev, A.I. Zelnikov, Nucl. Phys. B 486, 339 (1997).

V.P. Frolov, D.V. Fursaev, Phys. Rev. D 56, 2212 (1997).

[24] Y. Takahashi and H. Umezawa, Int. J. Mod. Phys. B 10, 1755 (1996) (reprinted from Collect. Phenomen. 2, 55 (1975)).

H. Umezawa, H. Matsumoto, M. Tachiki, Thermo Field Dynamics and Condensed States, North Holland, 1982.

[25] A. Perelomov, Generalized Coherent States and Their Applications, Springer, Berlin, Heidelberg, 1986.

[26] F. Strocchi, Elements of Quantum Mechanics of Infinite Systems, World Scientific, 1985. N.N. Bogolubov, A.A. Logunov, A.I. Oksak, and I.T. Todorov, General Principles of Quantum Field Theory, Kluwer Academic Publishers, Dordrecht, 1990.

[27] He-S. Song, D. Mi, L. Zhou, and C. Li, Chin. Phys. Lett. 19, 53 (2002).

He-S. Song, D. Mi, L. Zhou, and C. Li, Quantum Entanglement of Photons in Doubled q-Fock Space, quant-ph/0103024.

[28] I.S. Gradshteyn and I.M. Ryzhik, Table of Integrals, Series and Products, Fifth Edition Academic Press, San Diego, CA, 1994.

[29] R. Laflamme, Phys. Lett. B 196 (1987) 449. 